ORIGINAL ARTICLE

\title{
Evaluation of the Simplify D-dimer assay as a screening test for the diagnosis of deep vein thrombosis in an emergency department
}

\author{
D Neale, C Tovey, A Vali, S Davies, K Myers, M Obiako, V Ramkumar, A Hafiz
}

Emerg Med J 2004;21:663-666. doi: 10.1136/emj.2003.011049

See end of article for authors' affiliations .....................

Correspondence to: Mr C Tovey, Emergency Department, Prince Charles Hospital, Merthyr Tydfil, Mid Glamorgan, Wales CF47 9DT, UK; clive.tovey@nglam-tr. wales.nhs.uk

Accepted for publication 5 February 2004
Objectives: To evaluate the use in an emergency department of a new D-dimer assay (Simplify D-dimer) as a screening test for deep vein thrombosis (DVT).

Methods: 187 outpatients with clinical features suspicious of acute DVT were entered into this study. A Simplify D-dimer test was performed in the emergency department on all patients. A SimpliRED D-dimer test and a semi-automated latex agglutination assay (Auto-D-dimer 700 on a Thromboscreen 400C analyser) were performed in the haematology laboratory. All patients were investigated with contrast venography to confirm or exclude the diagnosis of DVT.

Results: The Simplify test had a sensitivity of $94.1 \%$ and a negative predictive value (NPV) of $94.8 \%$. These results compared favourably with the SimpliRED test (sensitivity 74.5\%, NPV $89.7 \%$ ) and the latex agglutination assay (sensitivity 90.2\%, NPV 92.2\%). This increased sensitivity was at the cost of a lower specificity, the specificity of the three D-dimer tests being Simplify $40.4 \%$, SimpliRED $83.1 \%$, and latex agglutination $43.4 \%$.

Conclusions: Simplify proved to be a rapid and easy to use test and may be useful for use in the emergency department as part of a diagnostic algorithm for deep vein thrombosis. Further larger scale studies are needed.
V nous thromboembolic disease has an estimated annual incidence in developed countries of 1 in 1000 people. ${ }^{1}$ The clinical diagnosis of deep vein thrombosis (DVT) of the lower limb is unreliable and individual symptoms and signs are of little value. ${ }^{23}$

Contrast venography has traditionally been the "gold standard" for the diagnosis of DVT $^{4}$ but venography has been replaced in most centres by colour flow duplex ultrasonography. Venography and ultrasonography are comparatively expensive investigations and over $75 \%$ of the investigated patients will prove not to have a DVT. ${ }^{56}$ Venography is also invasive and associated with a small but significant risk of complications. ${ }^{4}$

A screening test is needed with a high negative predictive value (NPV), which can thus be used to reduce the need for imaging. A fast, reliable, non-invasive, and inexpensive screening test is needed that can be performed immediately on attendance at hospital. In recent years interest has focused on the use of D-dimer assays and plethysmography. ${ }^{7}$

D-dimers are specific cross linked derivatives of fibrin, produced when fibrin is degraded by plasmin, so concentrations are raised in patients with venous thrombosis. ${ }^{8} \mathrm{D}$-dimer tests may be negative in cases of proven acute DVT for a number of reasons:

- D-dimer assays use a monoclonal antibody against the epitope on the D-dimer fragment that is absent on fibrin, fibrinogen, and non-cross linked fragments of fibrin. The reactivity of different $\mathrm{D}$-dimer monoclonal antibodies to these different molecular weight species varies, causing variable results with different assays within individual patients. ${ }^{9} 10$

- In isolated calf vein DVT the thrombus load will be less than in extensive proximal DVT. Patients with isolated calf vein DVT will therefore be expected to have lower plasma
D-dimer concentrations and this has been shown in some studies. ${ }^{11} 12$

- In the occasional patient there may be impaired fibrinolytic activity. ${ }^{13}$

- With the SimpliRED test there is a visual end point of agglutination of red blood cells and some studies have shown that interobserver variability in test results is high. $^{14} 15$

D-dimer tests generally have a high NPV but cannot be used in isolation as screening tests. D-dimer tests are therefore often used in conjunction with clinical probability scoring, plethysmography, or ultrasonography to reduce the need for further imaging. ${ }^{16-20}$ Although sensitive for venous thrombosis, high concentrations of D-dimer are insufficiently specific for making a positive diagnosis because they occur in other disorders such as malignancy, pregnancy, inflammatory conditions, and after surgery.

There are four types of D-dimer assays commercially available-enzyme linked immunosorbent assays (ELISA), latex agglutination assays, a whole blood agglutination assay (SimpliRED), and an immunochromatography test (Simplify). The SimpliRED and Simplify tests are suitable for use as bedside tests whereas the ELISA and latex agglutination tests need to be performed in a laboratory.

There are many quantitative latex agglutination and ELISA tests available ${ }^{91021}$ and the conventional ELISA is considered to be the gold standard for determination of D-dimer concentration. However, these laboratory based methods have the disadvantage that there can be a considerable delay in receiving the results in the emergency department.

Abbreviations: DVT, deep vein thrombosis; ELISA, enzyme linked immunosorbent assay; NPV, negative predictive value; PPV, positive predictive value 


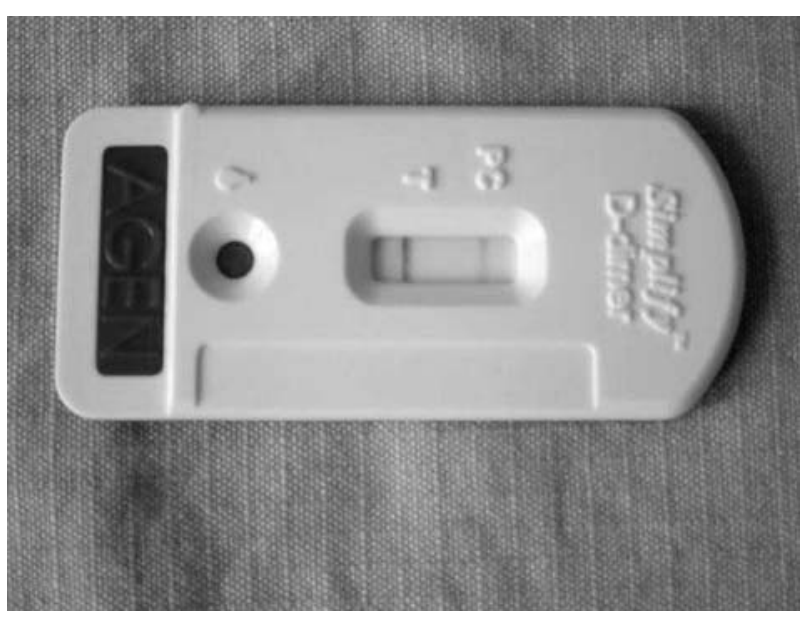

Figure 1 Photograph of a positive Simplify D-dimer test with a line visible in the test zone. A colour version of the figure is available on the journal web site (http://www.emjonline.com/supplemental).

Moreover, the laboratory based methods are more suited to the assay of many samples in one batch rather than individual samples performed as soon as the patient attends the emergency department. A number of rapid D-dimer assays are now available and include Tinaquant, Liatest, and Vidas D-dimer. These tests have sensitivities similar to conventional ELISA tests. ${ }^{21}$

The SimpliRED D-dimer test has been used widely as a near patient test in emergency departments and provides a result within five minutes. However, the sensitivity of the SimpliRED test has been reported to vary from $61 \%$ to $100 \%{ }^{8}$

A new near patient assay (Simplify D-dimer) has recently been introduced but there are currently no publications that have evaluated its efficacy. The aim of this study was to evaluate the use in the emergency department of the Simplify D-dimer test. The Simplify D-dimer test was compared with the (near patient) SimpliRED D-dimer assay and a (laboratory based) latex agglutination assay (Auto- D-dimer 700 on a Thromboscreen 400C analyser).

\section{METHODS}

The subjects were recruited into the study over a 21 month period starting in April 2001. The study was approved by the Bro-Taf Health Authority Local Research Ethics Committee and the subjects all gave informed written consent.

Patients presenting in the emergency department with clinical features suspicious of DVT were entered into the study, but the pre-test probability scores as performed in some studies $^{11}{ }^{18}$ were not calculated. Contrast venography was used to investigate DVT in all patients. As contrast venography is considered to be the gold standard, there was no follow up of patients with a negative venogram.

\begin{tabular}{llll}
$\begin{array}{l}\text { Table 1 } \\
\text { patients }\end{array}$ & D-dimer test and venogram results of the 187 \\
\hline \multicolumn{4}{c}{} \\
\end{tabular}

Patients were excluded from the study if they were less than 18 years old, had experienced recent trauma ( $<6$ weeks), had undergone recent surgery ( $<6$ weeks), were pregnant, had an underlying malignancy, or if they were having anticoagulant treatment. Patients were also excluded from the study if we were unable to perform venography (because of technical difficulties, or previous reaction to contrast).

Venous blood was obtained from each patient and a Simplify D-dimer test was performed immediately in the emergency department. Venous blood was also collected into plastic $4.5 \mathrm{ml}$ Tri-sodium citrate $(0.105 \mathrm{M})$ Becton-Dickinson vacutainers in a ratio of 9:1 (blood:anticoagulant) and sent to the haematology laboratory for assessing with the SimpliRED and latex agglutination tests.

\section{Simplify D-dimer}

The Simplify D-dimer assay consists of an individual test device in which the active ingredients are murine monoclonal antibody (DD3B6/22) specific for D-dimer conjugated to colloidal gold particles, a second D-dimer specific murine monoclonal antibody, and a sheep antimurine IgG antibody. Some $35 \mu \mathrm{l}$ of whole blood is added to the sample well followed by two drops of buffer. The device was left lying flat for 10 minutes and then checked for a pink/purple line in the procedural control zone of the reading window. The absence of a line in this zone renders the test invalid. A positive result was indicated by the presence of a pink/purple line in the test zone of the reading window indicating a D-dimer concentration of greater than $80 \mathrm{ng} / \mathrm{ml}$. A negative result was indicated by a complete absence of a line in the test zone of the reading window.

\section{SimpliRED D-dimer}

The SimpliRED D-dimer tests were performed in the haematology laboratory on the citrated whole blood. The methodology for the SimpliRED D-dimer assay has been described elsewhere. ${ }^{22}$ The assay contains a bispecific antibody that reacts with high affinity to D-dimer (3B6/22), and with a red cell binding antibody (RAT-1C3/86). In the presence of D-dimer concentrations $>120 \mathrm{ng} / \mathrm{ml}$, the antibody induces agglutination of the patient's red blood cells.

\section{Latex agglutination test}

After centrifugation of the citrated venous blood at 2000$3000 \mathrm{~g}$ for 10 minutes, the plasma was then separated and frozen at $-20^{\circ} \mathrm{C}$ for a maximum of one month. These samples were batch analysed for D-dimer using the Auto D-dimer (700) kit on the Thromboscreen 400C analyser.

The Auto D-dimer (700) is a quantitative latex microparticle enhanced turbidimetric immunoassay that uses monoclonal antibody coated (MA-8D3) latex particles. In the presence of D-dimer, the particles aggregate thus increasing turbidity. The increase in scattered light is proportional to the amount of D-dimer in the sample.

The turbidity change in the sample is measured on a Thromboscreen 400C photometer that is set to measure at $705 \mathrm{~nm}$. Photometric detection is started when the latex reagent is added to a cuvette containing the patient's sample. The change in optical density ( $\mathrm{OD}=$ Extinction) is monitored by the instrument. The turning point of the reaction curve is the final result and is displayed as $\mathrm{ng} / \mathrm{ml}$. The upper limit of the normal range was $120 \mathrm{ng} / \mathrm{ml}$.

To avoid any bias, the haematology staff were unaware of the venogram results. The radiology staff performing the venograms were also blinded to the D-dimer results. The emergency department performed the Simplify D-dimer test before the venogram. 
Table 2 Sensitivity, specificity, NPV, and PPV of the investigated D-dimer assays

\begin{tabular}{lllll}
\hline Test & Sensitivity \% & Specificity \% & NPV \% & PPV \% \\
\hline Simplify & $94.1(87.7$ to 100$)$ & $40.4(32.2$ to 48.7$)$ & $94.8(91.0$ to 98.6$)$ & $37.2(28.9$ to 45.6$)$ \\
SimpliRED & $74.5(62.5$ to 86.5$)$ & $83.1(76.8$ to 89.4$)$ & $89.7(82.0$ to 97.3$)$ & $62.3(50.1$ to 74.5$)$ \\
Auto D-dimer & $90.2(82.0$ to 98.4$)$ & $43.4(35.1$ to 51.7$)$ & $92.2(87.4$ to 96.9$)$ & $37.4(28.8$ to 45.9$)$ \\
\hline \multicolumn{4}{l}{ The figures in parentheses are the 95\% confidence intervals. } \\
\hline
\end{tabular}

The sensitivity, specificity, positive predictive value (PPV), NPV, and 95\% confidence intervals (CI) were calculated.

\section{RESULTS}

A total of 187 patients were enrolled into this study, 86 men and 101 women. Table 1 summarises the results and table 2 lists the sensitivity, specificity, NPV, and PPV for each of the D-dimer tests.

\section{DISCUSSION}

In this study $51(27.2 \%)$ patients had a positive venogram and the Simplify D-dimer test proved to have the highest sensitivity of the three methods (table 2). The Simplify test "missed" three cases of DVT. In one of the cases (a 66 year old woman) with calf vein DVT and a false negative Simplify test both the latex agglutination and SimpliRED tests were also negative. Two male patients (aged 39 and 80) with proximal DVT also had a false negative Simplify test. In both these cases the SimpliRED and latex agglutination tests were both positive. The latex agglutination test had five false negative results and the SimpliRED test 13 false negative results.

With any new D-dimer test it is mandatory to have a high sensitivity and to have few false negative D-dimer results. With the SimpliRED test there is a visual end point of agglutination of red blood cells. It is much easier to interpret the Simplify test as the positive end point is a pink/purple line. It is expected that the interobserver variability will be less with the Simplify test than the SimpliRED test.

With quantitative D-dimer assays it is important to select a cut off level such that the D-dimer assay has a high sensitivity. However, the cut off level should not be so low that the specificity of the assay is also very low. A cut off level must be chosen such that there is a balance between sensitivity and specificity. The upper limit of the normal Ddimer range was initially chosen to be $120 \mathrm{ng} / \mathrm{ml}$ for the latex agglutination test (based on a normal cohort of laboratory staff). The five false negative D-dimer results were $70 \mathrm{ng} / \mathrm{ml}$ or less. Unless the cut off level was changed to less than $70 \mathrm{ng} / \mathrm{ml}$ the sensitivity of the assay would not be improved and there would be a very low specificity at a cut off level of $70 \mathrm{ng} / \mathrm{ml}$.

It has been reported that there is a significantly lower sensitivity for the SimpliRED test if citrated whole blood is used instead of fingerstick capillary samples. ${ }^{15}$ Although the manufacturers of SimpliRED suggest that fingerstick capillary blood can be used, in the emergency department it is usually easier to take venous blood, as other blood investigations often need to be performed. In this study we used citrated venous blood for the SimpliRED test because the sample was sent to the haematology laboratory. The Simplify test was performed on venous blood.

The increased sensitivity of the Simplify D-dimer test when compared with the other two tests was at the expense of specificity (table 2 ). The specificity of the Simplify test was only $40.4 \%$, compared with SimpliRED (83.1\%) and latex agglutination $(43.4 \%)$.

\section{CONCLUSION}

The Simplify D-dimer is easy to use, can be performed in 10 minutes, and the sensitivity was $94.1 \%$ in this study. This test is more suitable for use in the emergency department than the SimpliRED test. However, despite the high NPV of $94.8 \%$, this test should be used in conjunction with clinical probability scoring, plethysmography, or ultrasonography to reach failure rates of less than $1 \%$.

The Simplify test costs about $£ 7$, which is similar to most Ddimer tests. However, the laboratory based tests also need an analyser that entails a considerable initial capital cost. The cost of contrast for venography is on average over $£ 30$ and more importantly, ultrasonography and venography are very time consuming for skilled hospital staff. Despite the low specificity of the Simplify test, any reduction in further imaging would make the test worthwhile from an economic viewpoint.

\section{CONTRIBUTORS}

DN drafted the original manuscript, did the statistical analysis, and the laboratory investigations. CT initiated and coordinated the study, examined the subjects, and did the Simplify tests. AV was the radiology lead for the study and performed many of the venograms. SD did many of the laboratory investigations. KM was the haematology lead for the study. MO, VR, and AH examined the subjects and did the Simplify tests. All authors contributed to the final draft of the paper. CT is the guarantor.

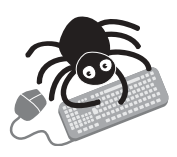

A colour version of the figure is available on the journal web site (http://www.emjonline.com/ supplemental).

\section{Authors' affiliations}

D Neale, S Davies, K Myers, Haematology Department, Prince Charles Hospital, Merthyr Tydfil, Mid Glamorgan, Wales

C Tovey, M Obiako, V Ramkumar, A Hafiz, Emergency Department,

Prince Charles Hospital

A Vali, Radiology Department, Prince Charles Hospital

Funding: none.

Competing interests: none declared.

\section{REFERENCES}

1 Carter CJ. Epidemiology of venous thromboembolism. In: Hull R, Pineo GF eds. Disorders of thrombosis. Philadelphia: WB Saunders, 1996:159-74.

2 Ebell MH. Evaluation of the patient with suspected deep vein thrombosis. J Fam Pract 2001;50:167-71.

3 O'Donnell TF Jr, Abbott WM, Athanasoulis CA, et al. Surg Gynecol Obstetr 1980;150:69-74.

4 deValois JC, van Schaik CC, Verzijlbergen F, et al. Contrast venography: from gold standard to 'golden backup' in clinically suspected deep vein thrombosis. Eur J Radiol 1990;11:131-7.

5 Heijboer $\mathbf{H}$, Büller HR, Lensing AWA, et al. A comparison of real-time compression ultrasonography with impedance plethysmography for the diagnosis of deep-vein thrombosis in symptomatic outpatients. N Engl J Med 1993;329:1365-9.

6 Cogo A, Lensing AWA, Koopman MMW, et al. Compression ultrasonography for diagnostic management of patients with clinically suspected deep vein thrombosis: prospective cohort study. BMJ 1998;316:17-20.

7 Tovey C, Wyatt S. Diagnosis, investigation, and management of deep vein thrombosis. BMJ 2003;326:1180-4. 
8 Kelly J, Rudd A, Lewis RR, et al. Plasma D-dimers in the diagnosis of venous thromboembolism. Arch Intern Med 2002;162:747-56.

9 Schutgens REG, Haas FJLM, Gerritsen WBM, et al. The usefulness of five $D$-dimer assays in the exclusion of deep venous thrombosis. Journal of Thrombosis and Haemostasis 2003;1:976-81.

10 Dempfle CE, Zips S, Ergül H, et al. The fibrin assay comparison trial (FACT): evaluation of 23 quantitative $D$-dimer assays as basis for the development of D-dimer calibrators. Thromb Haemost 2001;85:671-8.

11 Lennox AF, Delis KT, Serunkuma S, et al. Combination of a clinical risk assessment score and rapid whole blood D-dimer testing in the diagnosis of deep vein thrombosis in symptomatic patients. J Vasc Surg 1999;30:794-803.

12 Chapman CS, Akhtar N, Campbell S, et al. The use of D-dimer assay by enzyme immunoassay and latex agglutination techniques in the diagnosis of deep vein thrombosis. Clin Lab Haematol 1990;12:37-42.

13 Eisenberg PR. Does a negative D-dimer exclude thrombosis? Fibrinolysis 1993;7(suppl 2):32-5.

14 Turkstra F, van Beek EJR, Büller HR. Observer and biological variation of a rapid whole blood D-dimer test. Thromb Haemost 1998;79:91-3.

15 Mauron T, Baumgartner I, Z'Brun A, et al. SimpliRED D-dimer assay: comparability of capillary and citrated venous whole blood, between-assay variability, and performance of the test for exclusion of deep vein thrombosis in symptomatic outpatients. Thromb Haemost 1998;79:1217-19.

16 American Thoracic Society. The diagnostic approach to acute venous thromboembolism. Am J Respir Crit Care Med 1999;160:1043-66.

17 Kraaijenhagen RA, Lensing AWA, Wallis JW, et al. Diagnostic management of venous thromboembolism. Ballieres Clin Haematol 1998; 11:541-85.

18 Aschwanden $M$, Labs $\mathrm{KH}$, Jeanneret C, et al. The value of rapid D-dimer testing combined with structured clinical evaluation for the diagnosis of deep vein thrombosis. J Vasc Surg 1999;30:929-35.

19 Kearon C, Ginsberg JS, Douketis J, et al. Management of suspected deep venous thrombosis in outpatients by using clinical assessment and D-dimer testing. Ann Intern Med 2001;135:108-11.

20 Ginsberg JS, Kearon C, Douketis J, et al. The use of D-dimer testing and impedance plethysmographic examination in patients with clinical indications of deep vein thrombosis. Arch Intern Med 1997;157:1077-81.

21 van der Graaf $F$, van den Borne $H$, van der Kolk M, et al. Exclusion of deep vein thrombosis with $\mathrm{D}$-dimer testing. Comparison of $13 \mathrm{D}$-dimer methods in 99 outpatients suspected of deep venous thrombosis using venography as reference standard. Thromb Haemost 2000;83:191-8.

22 John MA, Elms MJ, O'Reilly EJ, et al. The SimpliRED D-dimer test: a novel assay for the detection of cross-linked fibrin degradation products in whole blood. Thromb Res 1990;58:273-81.

\section{CONTINUING MEDICAL EDUCATION}

\section{ACCESS: the acute cerebral CT evaluation stroke study}

$\mathrm{D}$

you interpret CT brain scans within the first few hours of stroke? We run the acute cerebral CT evaluation stroke study (ACCESS), an internet based, interactive, CT reading tool designed to evaluate and improve CT reader reliability in detecting early infarct sign on CT. Readers log on to a web server (http://www.neuroimage.co.uk), complete a few details about background training and experience in viewing CT scans, view the study CT scans, and answer questions about each scan on the same screen. It is our intention to investigate the cross disciplinary recognition of stroke and to improve upon pre-existing paradigms in the diagnosis of acute stroke on CT.

There are 56 scans, reviewed in batches of about 10 scans each of which take about 20 minutes to complete. We will present the results of the study once it is completed later this year. Participation in ACCESS counts towards five continuing medical education category 1 credits in the UK. Accreditation was awarded by the Royal College of Radiologists, but because accreditation is not specific to RCR members, there is cross recognition between all UK Royal Colleges.

J Wardlaw

University of Edinburgh, Department of Clinical Neurosciences, Western General Hospital, Crewe Road, Edinburgh EH4 2XU, UK; jmw@skull.dcn.ed.ac.uk 